\title{
Cyclophosphamide Anhydrous
}

National Cancer Institute

\section{Source}

National Cancer Institute. Cyclophosphamide Anhydrous. NCI Thesaurus. Code C61694.

The anhydrous form of cyclophosphamide, a synthetic nitrogen mustard alkylating agent, with antineoplastic and immunosuppressive activities. In the liver,

cyclophosphamide is converted to active metabolites including phosphoramide mustard, which binds to and crosslinks DNA and RNA, thereby inhibiting DNA replication and protein synthesis. This agent, at low doses, is also a potent immunosuppressant primarily by depleting T-regulatory cells. 